\title{
State of stress in piezoelectric elements with interdigitated electrodes
}

\author{
Rolf Paradies • Mark M. Melnykowycz
}

Received: 1 February 2008 /Accepted: 14 August 2008 /Published online: 12 September 2008

(C) Springer Science + Business Media, LLC 2008

\begin{abstract}
Finite element analyses have been performed on the representative volume element of piezoelectric elements with interdigitated electrodes (IDE). In these investigations the change of the piezoelectric material properties during polarization has been considered prior to additional service loads, e.g. mechanical loading. The numerical results obtained with a specially coded finite element routine are compared with a reference model given by the so called uniform field model. The model indicates stress concentrations at the tip of the finger electrode, which have been validated by experimental results. The modeling approach presented allows for a better understanding of the overall effects in piezoelectric materials with IDE.
\end{abstract}

Keywords Piezoelectric · Interdigitated - Electrode · Finite element analysis $\cdot$ Stress $\cdot$ Experiment

\section{Introduction}

One dominant group of solid state actuator and sensor elements are piezoelectric transducers. They are easily controlled by applying an electric field to the element, resulting in a mechanical response. While non-polarized material exhibits mechanically isotropic material properties in the absence of global piezoelectric polarization the

Rolf Paradies' e-mail will only be available until the end of the year. For any inquiries, you may contact Michel Barbezat at Michel.

Barbezat@empa.ch and at http://www.empa.ch/plugin/template/empa/ $* / 15179 /$ - $/ 1=1$.

R. Paradies $(\varangle) \cdot$ M. M. Melnykowycz

Laboratory for Mechanical Systems Engineering,

Empa-Materials Science and Technology,

Ueberlandstrasse 129,

8600 Dübendorf, Switzerland

e-mail: rolf.paradies@empa.ch material properties become transversally isotropic with respect to the polarization direction after polarization. The link between material property values and polarization direction in piezoelectric materials has been shown by Tanimoto et al. [1], where polarization along and perpendicular to the direction of loading was seen to modify the tensile strength of polarized, as compared with non-polarized lead zirconate titanate (PZT) and $\mathrm{BATiO}_{3}$ tensile specimens. Further research showed that crack propagation behavior is dependent on polarization direction in PZT wafers [2]. Therefore, the characterization and modeling of the link between materials properties and polarization character is important to investigate, given the demonstrated susceptibility of damage evolution near the electrodes of PZT actuators as investigated by Ru et al. [3] and Ye [4].

The scope of the current investigation is to point out the influence of the inhomogeneous electric field distribution on the material properties of piezoelectric elements with interdigitated electrodes (IDE) and to present a modeling approach for more precise calculations within the representative volume element (RVE).

\section{Models for piezoelectric elements with IDE}

In addition to monolithic dense ceramic wafers, piezoelectric composites are widely used due to their higher reliability with respect to fracture toughness. This is accomplished by embedding uniaxially aligned piezoceramic fibers in a polymer matrix before placing them between two sets of IDE. This arrangement results in a highly orthotropic transducer element with larger flexibility perpendicular to the working direction compared to a monolithic piezoelectric wafer. The best know representatives of this group are the active fiber composite (AFC) [5] and the Macro Fiber Composite (MFC) [6]. 
One analytical model which can be used for describing the electromechanical coupling of piezoelectric materials as well as piezoelectric composites with IDE is the uniform field model (UFM) by Bent [5]. It is based on the smallest volume possible which represents the properties of the complete element. This relevant region named the RVE is shown in Figs. 1 and 2. The RVE covers one half of the thickness of an AFC/MFC and stretches in the fiber direction from the middle of one finger electrode half way to the middle of the adjacent finger electrode. Likewise, it reaches from the middle of a fiber half way to the middle of the adjacent fiber, perpendicular to the fiber plane. In the principal assumption of the UFM each RVE is composed of three regions, where each region has homogenous properties, which are either arranged in pairs parallel or in series, respectively. The regions named Cases A, B and C capture the electromechanical properties in the area between two finger electrodes of different electric potential and, thereby, allow the calculation of the piezoelectric properties of the RVE. As implicated in the name UFM, an electrical field of uniform strength is assumed within each region representing a homogenous polarization in the same direction. This assumption is not valid in the vicinity of the PZT-electrode interface where there is a gradual change in the electric field (Fig. 2 actual geometry and Fig. 1 cut-out). This effect is not covered in the UFM due to its geometrical complexity and can be neglected in the description of the total strain of the RVE which is the main goal of the UFM-solution. Likewise, the change in the material properties in the vicinity of the electrode due to the same change in the polarizing electric field is not covered in the UFM for the same reason. In the case of a stress

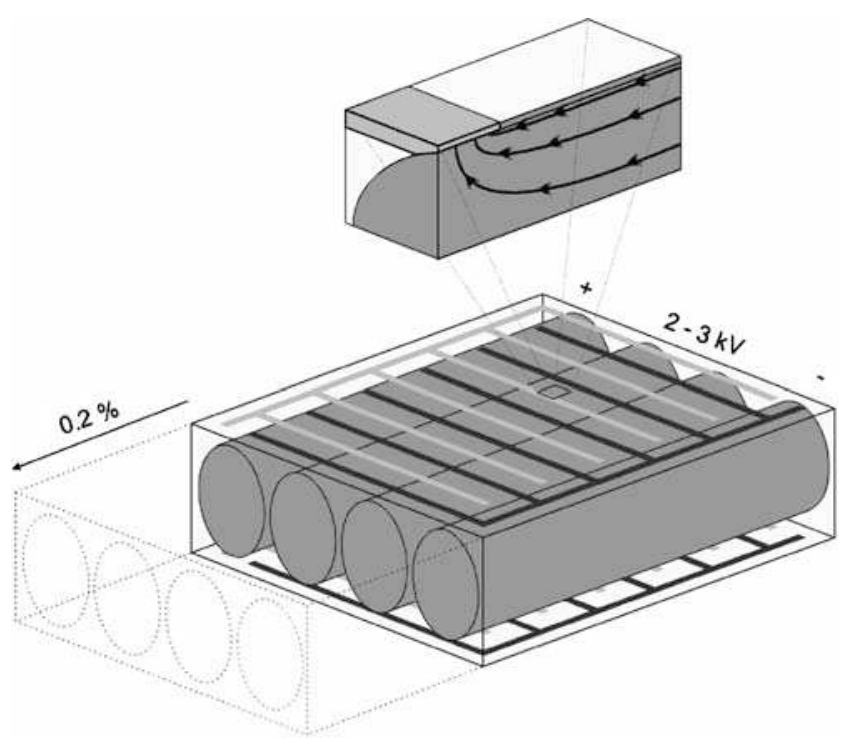

Fig. 1 Sketch of an AFC with IDE and cut-out representing the RVE plus indicated electric field orientation [7]

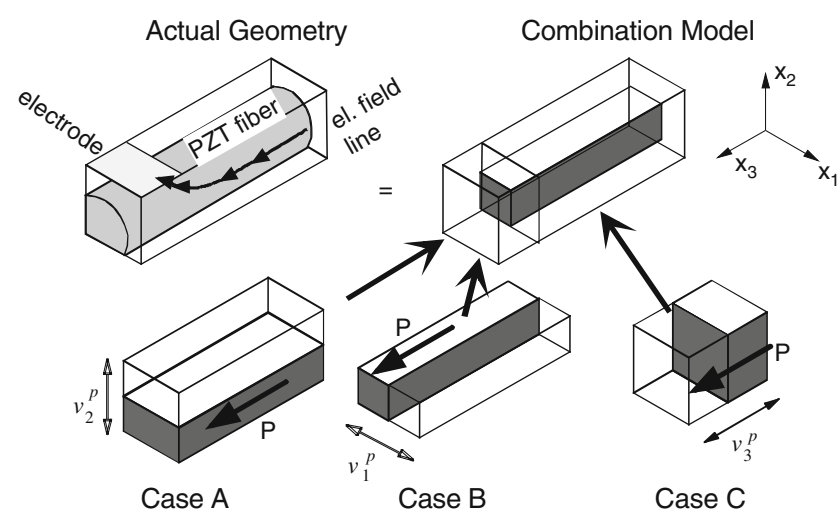

Fig. 2 The UFM introduced by [5] with the polarization vector P and the fraction of piezoelectric material within each phase

analysis both effects have to be considered. The current work investigates the influence of the material properties on the state of stress within the RVE and thereby of the transducer element.

\section{FE-investigation of AFC}

Published FE-investigations related to piezoelectric transducer elements describe mainly the strain behavior within the RVE e.g. [8-10] and thereby resemble the UFM of the RVE. The main issue for finite element simulations is to ensure a correct representation of the structure, boundary conditions and the material properties. While geometry and boundary conditions of a RVE can be easily specified, problems arise in the correct assignment of the material properties. This is due to the gradual change of the PZT material properties in relation to the same change in the electric field. At the electrode, the electric field lines are perpendicular to the plane of the IDE while they are parallel between two electrodes of different electric potentials (cp. Figs. 1 and 2). Furthermore, the electric field lines concentrate near the electrode and thin out in other areas, resulting in an inhomogeneous field distribution and thereby a variation in the material properties of the polarized piezoelectric fibers.

The following discussion will focus on the problems which arise during stress calculations within piezoelectric materials with IDE. This investigation is based on AFC manufactured at Empa (Fig. 3 with the material properties given in Table 1). The material properties of the polarized PZT-5A are based on datasheets. Although several publications are available with elastic properties of non-poled barium titanite $[11,12]$ no literature could be found with the complete material description of the non-polarized PZT5A. Therefore, those stiffness properties have been derived from [13] assuming an isotropic material behavior while the 
Fig. 3 Sketch of RVE with dimension for AFCs manufactured at Empa

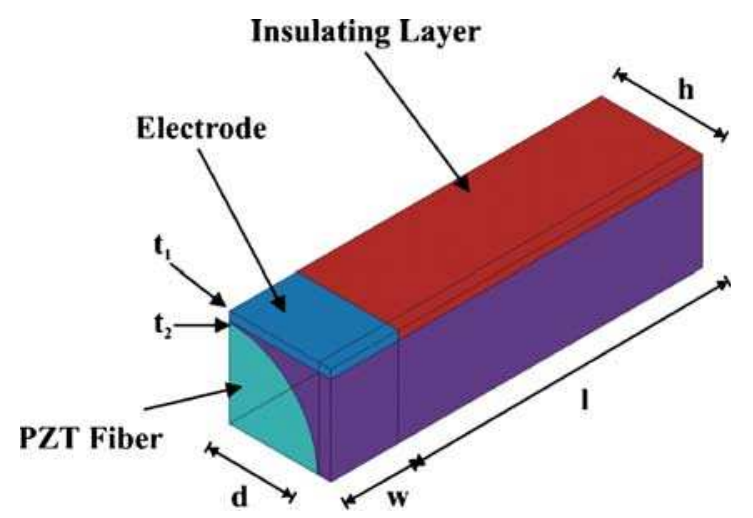

1: $900 \mu \mathrm{m}$

Width of finger electrode:

$\mathrm{w}: 200 \mu \mathrm{m}$

Diameter of PZT fiber:

Fiber spacing:

Height of insulating layer:

Fiber-electrode distance: d: $260 \mu \mathrm{m}$

h: $300 \mu \mathrm{m}$

$\mathrm{t}_{1}: 13 \mu \mathrm{m}$

$\mathrm{t}_{2}: 2 \mu \mathrm{m}$ charge coefficients have been set to zero. The change in the relative permittivity is based on capacity measurements of poled and non-poled PZT-wafers at Empa. The numerical simulations were performed with ANSYS Release 11.0 using two and three dimensional models (2D-FE-model, 3D-FE-model) with plane and solid coupled field elements for representing the piezoelectric behavior (PLANE223, SOLID226). The fiber are embedded in an epoxy matrix with the material properties: $E=3 \mathrm{GPa}, v=0.3$ and $\varepsilon_{\mathrm{r}}=3.4$.

\subsection{Material description within the RVE of a transducer} with IDE

The material properties of a piezoelectric material vary with its state of polarization. In case of a piezoelectric element with IDE, there is a gradient of different material properties (e.g. non-polarized, polarized). Furthermore, there are different orientations of the material coordinate systems, which have to be described for stress calculations of such elements. This description can be achieved by

Table 1 Material properties used for the FE-investigation.

\begin{tabular}{llll}
\hline Material properties/description & $\begin{array}{l}\text { Polarized } \\
\text { PZT-5A }^{\mathrm{a}}\end{array}$ & $\begin{array}{l}\text { Non-polarized } \\
\text { PZT }\end{array}$ & Units \\
\hline$\rho$ & 7,500 & 7,500 & $\mathrm{~kg} / \mathrm{m}^{3}$ \\
$\mathrm{~d} / \mathrm{V}$ \\
$\mathrm{d}_{31}$ & $-1.71 \mathrm{E}-10$ & - & $\mathrm{m} / \mathrm{V}$ \\
$\mathrm{d}_{33}$ & $3.74 \mathrm{E}-10$ & - & $\mathrm{m} / \mathrm{V}$ \\
$\mathrm{d}_{15}$ & $5.84 \mathrm{E}-10$ & - & $\mathrm{m} \mathrm{s} / \mathrm{kg}$ \\
$s_{11}^{E}$ & $1.64 \mathrm{E}-11$ & $1.53 \mathrm{E}-11$ & $\mathrm{~m} \mathrm{~s}^{2} / \mathrm{kg}$ \\
$s_{33}^{E}$ & $1.88 \mathrm{E}-11$ & $1.53 \mathrm{E}-11$ & $\mathrm{~m} \mathrm{~s}^{2} / \mathrm{kg}$ \\
$s_{12}^{E}$ & $-5.74 \mathrm{E}-12$ & $-4.59 \mathrm{E}-12$ & $\mathrm{~m} \mathrm{~s}^{2} / \mathrm{kg}$ \\
$s_{13}^{E}$ & $-7.22 \mathrm{E}-12$ & $-4.59 \mathrm{E}-12$ & $\mathrm{~m} \mathrm{~s}^{2} / \mathrm{kg}$ \\
$s_{44}^{E}$ & $4.75 \mathrm{E}-11$ & $3.98 \mathrm{E}-11$ & - \\
$\varepsilon_{11}^{T} / \varepsilon_{\mathrm{o}}$ & 1730 & $1000^{\mathrm{b}}$ & - \\
$\varepsilon_{33}^{\mathrm{T}} / \varepsilon_{\mathrm{o}}$ & 1700 & $1000^{\mathrm{b}}$ & - \\
\hline
\end{tabular}

${ }^{a}$ Source:http://www.efunda.com/materials/piezo/material_data/matdata_ output.cfm?Material_ID=PZT-5A

${ }^{\mathrm{b}}$ Based on capacity measurements of poled and non-poled PZT-wafers including the polarization process of the piezoelectric transducer into the FE-simulation. A corresponding procedure which allows for an automatic material assignment according to the electric field condition is shown in Fig. 4 $[7,14]$. For a completely given set of material properties this procedure allows for the numerical investigation of any piezoelectric transducer element for arbitrary electrode configurations.

Being able to provide the material properties as a function of the polarization field requires extensive experimental investigations, while a perfect alignment between material direction and electrical polarization field can be accomplished more easily in the FE-environment. Therefore, the influence of the material orientation on the overall properties of a piezoelectric transducer element has been considered first. Figure 5 shows the material orientation along the field lines of an electric field between two electrodes of an AFC obtained by the procedure described in Fig. 4). The plot depicts a cross section of a piezoelectric fiber between a pair of positive and negative finger electrodes (upper half of element). The material orientation is indicated by element coordinate systems (triad) while the electric field vector is represented by arrows. At the beginning of the simulation the material was defined as non-polarized and oriented parallel to the $\mathrm{x}$-axis in all finite elements. Within three steps of the iteration procedure the material is divided between a non-polarized (dark area) and a polarized region based on the defined poling threshold (Fig. 4). Furthermore, the poled material is orientated perfectly along the field lines indicating the two planes of symmetries within the FE-model, which represents four RVEs.

\subsection{Stress state within a RVE for the UFM and the new approach}

For the investigation of the effect of the material modeling on the stress state in the RVE of the AFC shown in Fig. 3 a corresponding FE-model consists of 2666 elements (Type 
Fig. 4 Flow-chart for stress evaluation in piezoelectric elements with IDE [7, 14]

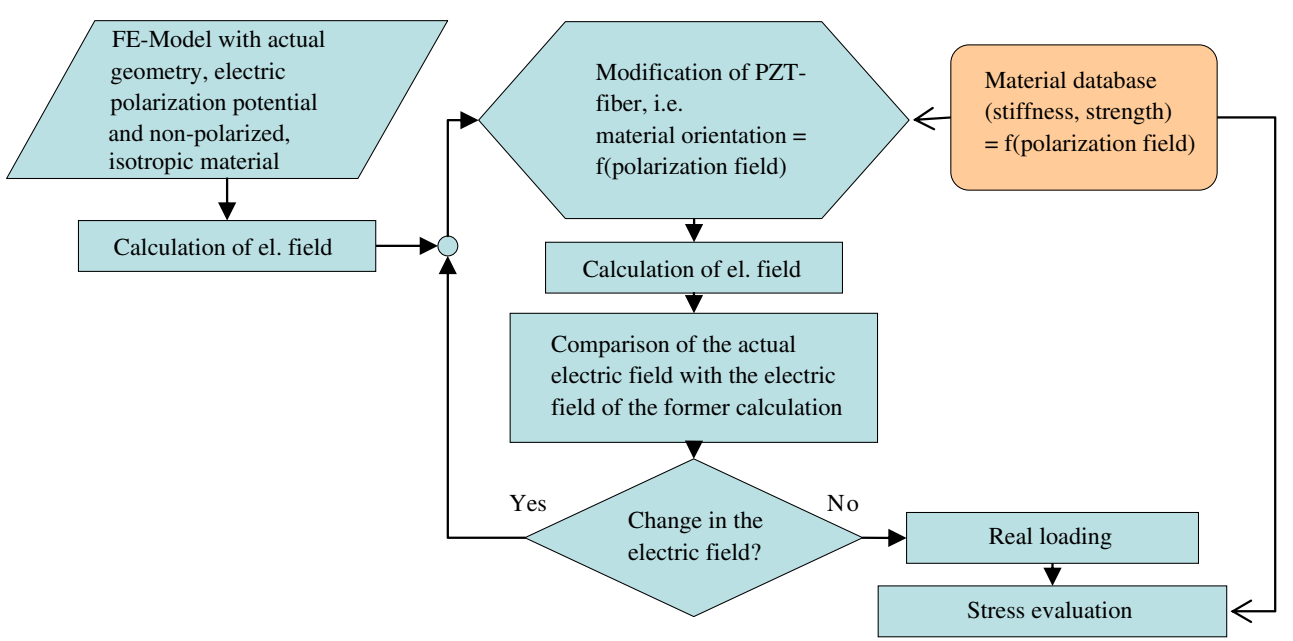

positive pole

negative pole

strength near left electrode edge $[\mathrm{V} / \mathrm{mm}](\mathbf{d})$

Fig. 5 Material distribution (a) and electric field lines between two electrodes (b) with enlarged view near the left electrode and with additional material orientation triads (c) electric field $[\mathrm{V} / \mathrm{mm}](\mathbf{d})$

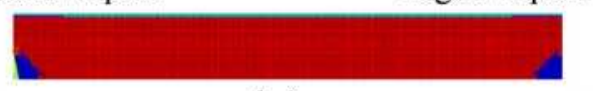

(a)

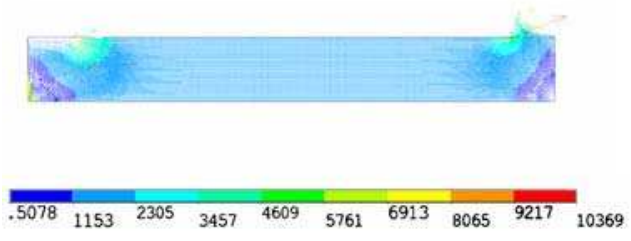

(c)

Fig. 6 3D-FE-model of RVE with piezoelectric material, matrix and finger electrode for UFM-assumption (a) without matrix (b)

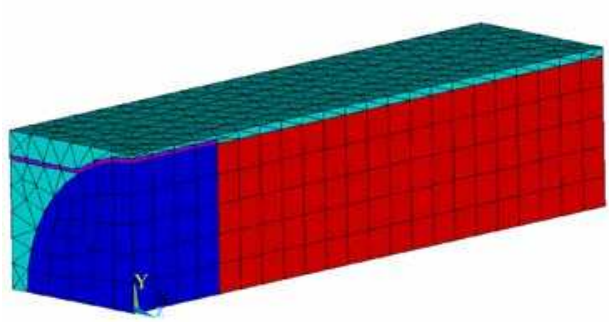

(a)

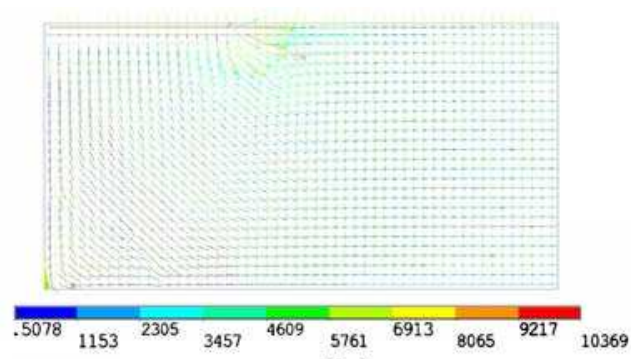

(b)

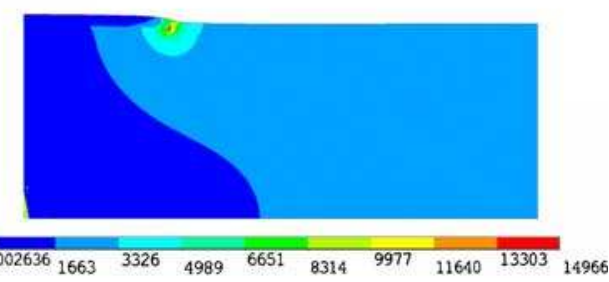

(d)

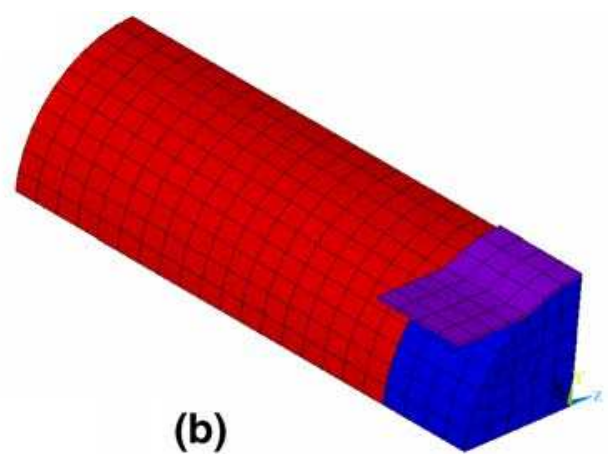



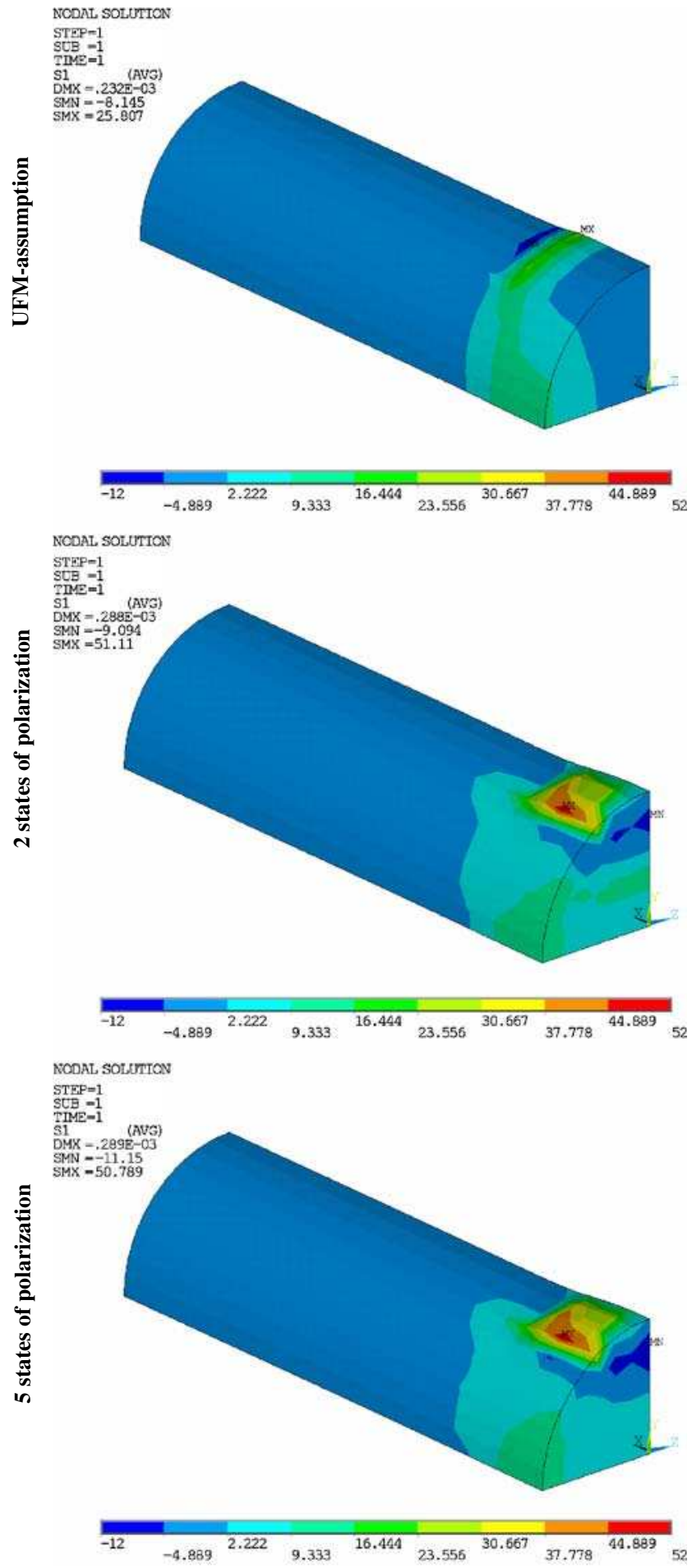

Fig. 7 First principal stress distribution $[\mathrm{MPa}]$ in RVE due to polarization

SOLID226) has been used. This model shown in Figs. 6 and 7 includes the piezoelectric material, the epoxy matrix, as well as the electrode and resembles a typical standard AFC manufactured at Empa. Three different material models and two load cases have been considered. The reference is given by the UFM-assumption of non polarized and perfectly polarized piezoelectric material below and between the finger electrodes. In addition poling code in Fig. 4 calculated automatically the piezoelectric material orientation and material distribution according to predefined poling levels for two and five different states of the piezoelectric material [15]. The two load cases investigated are an electrical load acting on the RVE and a tensile load acting in the fiber direction of the poled RVE with the boundary conditions (BCs) in Table 2 .

The results of this comparison are presented in Figs. 7 and 8, respectively, for three representations of the piezoelectric material. The results of this comparison are presented in Figs. 7 and 8, respectively, while the maximum stresses are summarized in Table 3. For a better comparison the same stress legend has been used for the three different FE-models in Fig. 7 (Principal stress distribution in RVE due to polarization) whereby stress values below the given range are colored in dark grey. The same approach has been used for the stress plots in Fig. 8 for the second load case (principal stress distribution in RVE due to tensile load after polarization).

In general, the simulation of the polarization process results in a lower stress state in the RVE compared to the UFM-assumption. An exception is the stress concentration near the finger electrode where the maximum stress values increases. This effect is even greater at the end of the electrode in the tangential direction of the piezoelectric fiber, which becomes clearly visible in Figs. 7 and 8. A possible remedy for this effect is the IDE of the polymeric AFC described in [16] which covers the piezoelectric fiber by $360^{\circ}$, thereby, avoiding a discontinuity in tangential direction.

\section{Experimental investigation}

In the absence of exact material data and missing strength criteria a detailed experimental verification of the presented numerical investigation is not possible. This would require tensile measurement of piezoelectric transducers with IDE and the comparison of the ultimate tensile stress obtained in

Table 2 BCs for FEM investigation of the material influence on the FE-results.

\begin{tabular}{lll}
\hline Location in FE-model & $\begin{array}{l}\text { Load case: } \\
\text { polarization }\end{array}$ & $\begin{array}{l}\text { Load case: tensile load } \\
\text { after polarization }\end{array}$ \\
\hline Left face: $[\mathrm{mm}]$ & $u_{\mathrm{x}}=0$ & $u_{\mathrm{x}}=0$ \\
Lower face: $[\mathrm{mm}]$ & $u_{\mathrm{y}}=0$ & $u_{\mathrm{y}}=0$ \\
Right face: $[\mathrm{mm}]$ & $d u_{x} / d_{x}=0$ & $u_{\mathrm{x}}=1.1 \mathrm{E}-3 \mathrm{~mm}=0.1 \%$ strain \\
Left electrode: $[\mathrm{kV}]$ & +1 & +1 \\
Right electrode: $[\mathrm{kV}]$ & -1 & -1 \\
\hline
\end{tabular}



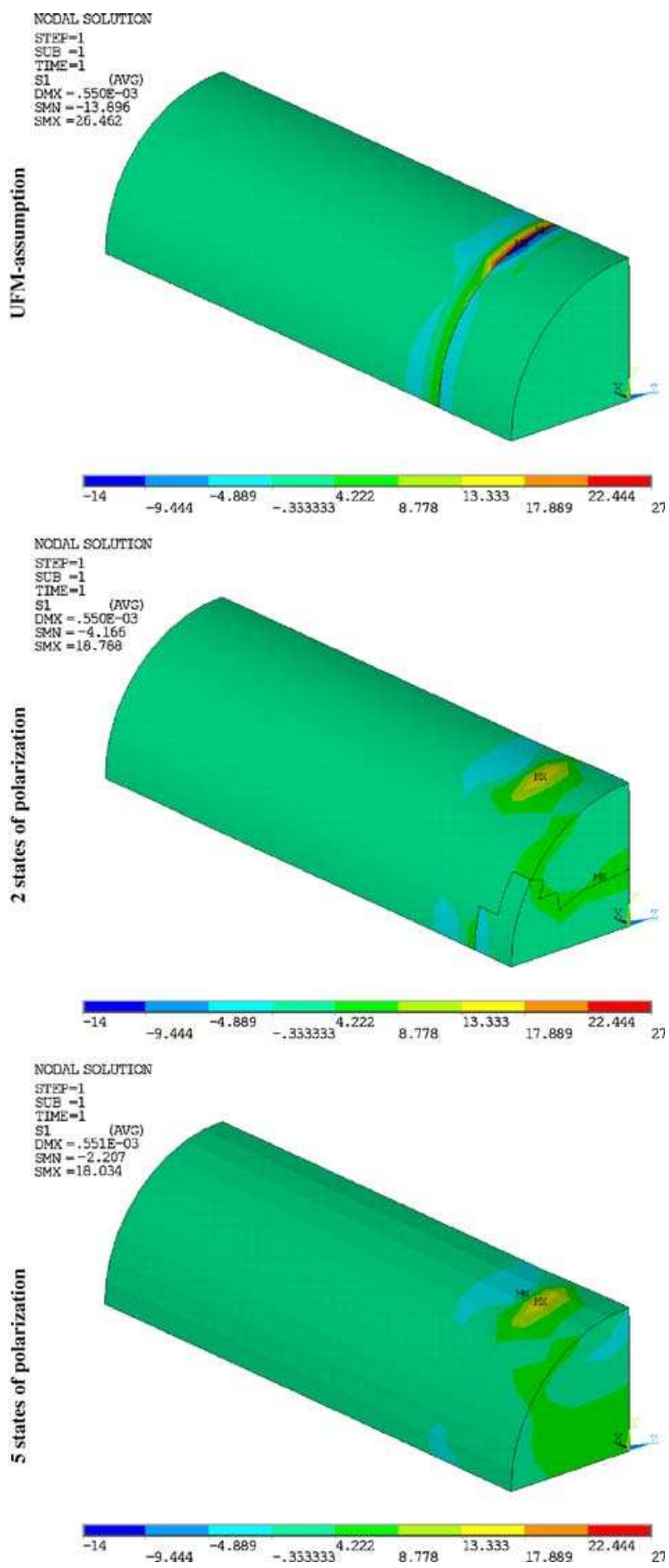

Fig. 8 First principal stress distribution [MPa] in RVE due to tensile load $(0.1 \%$ strain) after polarization

the test with the stress values found in the FE-model. In view of the dimensions of the RVE (cp. Fig. 3) local stress measurements are not possible. Therefore, measurement strategies have to be developed for assessing the validity of the numerical investigation. A first step in this direction is the validation of the effect in general, i.e. that given certain loading conditions, the state of polarization will increase the failure susceptibility of polarized versus non-polarized piezoelectric material and that the place of the failure is at the predicted stress concentrations at the edge of the finger electrode. The model presented predicts stress concentrations at the edge between the IDE finger and the fiber which are caused by the change in the material properties. This statement corresponds with literature e.g. [17]. Furthermore, the model shows no stress concentrations in non-polarized material far away from the IDE. These regions are not to be confused with the non-polarized areas directly under the finger electrode shown in the figures representing the RVE only (cp. Fig. 6).

For the validation of the numerical predictions presented, AFC made at Empa have been investigated with respect to their failure behavior. In a previous study polarized AFC were bonded to a glass fiber reinforced laminated substrate, and then subjected to tensile strains up to $0.50 \%$. The specimen was monitored during loading to identify the fragmentation behavior in the PZT fiber mat [7]. From these fragmented AFC polished micrograph sections have been taken, where the insulating foil (Kapton ${ }^{\circledR}$ ) and the printed IDE surface was stripped away from one side via mechanical grinding. Indirect lighting of the specimen surface was used to observe the exposed AFC surface and investigate cracking in the PZT fiber mat (cp. Fig. 9).

In the image from a fractured AFC specimen shown in Fig. 9, the dark horizontal region is the shadow of the bottom IDE finger, which establishes the position of the IDE finger relative to the fibers. Near the edge of the IDE finger sharp transitions are seen, which correspond to free surfaces in the fibers and thereby, indicate cracks running through the thickness of the fiber. Theses cracks reside at the edge of the IDE finger (see Fig. 9(b)), which coincides with the location of stress concentrations predicted in the FE model. A gradient of material properties, as related to the gradient in electrical field distribution during polarization in the vicinity of the IDE edge, would lead to stress concentrations, and consequentially a larger susceptibility to failure in those material regions. This discussion does not include that in areas of high stress, stress relief may occur due to domain motion. This behavior is not included - as can occur at crack tips.

In the right side of Fig. 9(a) there are few sharp transitions at the IDE fingers. This corresponds as well with the findings of the numerical investigation, since the piezoelectric material between those fingers of identical electric potential are not polarized. On the right side, the region between IDE fingers is uniform, without evidence of cracking along the IDE edge. This shows that the position of the IDE finger has a direct effect on crack development in the PZT fibers. Since the IDE pattern defines the electrical field distribution during polarization, it is evident 
Table 3 Stress results [MPa] for the investigated material properties and two load cases.

\begin{tabular}{|c|c|c|c|c|c|c|}
\hline Load case & Strength criteria & $\begin{array}{l}\text { Reference: } \\
\text { UFM-assumption }\end{array}$ & $\begin{array}{l}2 \text { States of } \\
\text { polarization }\end{array}$ & Difference $[\%]$ & 5 States of polarization & Difference $[\%]$ \\
\hline \multirow[t]{2}{*}{ Polarization } & 1st Principal stress & 26 & 51 & 96 & 51 & 96 \\
\hline & von Mises Stress & 70 & 39 & -44 & 39 & -44 \\
\hline \multirow[t]{2}{*}{ Tensile load after polarization } & 1st Principal stress & 26 & 19 & -27 & 18 & -31 \\
\hline & von Mises Stress & 153 & 103 & -33 & 101 & -34 \\
\hline
\end{tabular}

that the polarization state of the PZT fibers is related to the observed crack locations.

The experimental investigation presented in Fig. 9 clearly shows a correlation between IDE finger position and the location of cracks in the polarized fibers, as indicated in the FE model.

\section{Conclusion}

The material properties of piezoelectric materials strongly depend on their state of polarization. The influence of the material properties on the stress for a mechanical and an electrical load has been demonstrated. While the resulting effects of gradually changing material properties can be neglected for the calculation of the free actuation strain they should be considered with respect to stress evaluations within piezoelectric elements with IDE. This can be accomplished by modeling the polarization process of the element prior to the loading step. Such a simulation of the polarization process requires exact material data (e.g. strength, stiffness) for the piezoelectric material at different states of polarization as well as an automated procedure for the individual assignment of these data to the model. An appropriate procedure has been used for the investigation of the micromechanical effects within the RVE of piezoelectric transducer elements with IDE. The qualitative findings of this investigation have been validated by experimental tests while so far there are no experimental possibilities available for the quantitative validation of this theoretical approach. Therefore, further investigations have to focus on providing the material properties according to their polarization within the PZT-material. Especially, the determination of the required strength and stiffness values necessary for these strength predictions has to be investigated in further studies. The expected benefit of this procedure is a better understanding of the micromechanical effects in piezoelectric elements with complex electrode configurations, e.g. IDE and a more accurate prediction of the stress state within these elements which is important for the prediction of their fatigue behavior as well as the affect of new IDE designs.
Fig. 9 Section of fragmented AFC with (a) cracking locations identified in relation to the IDE finger position and (b) detail of cracked fibers in the vicinity of a finger electrode
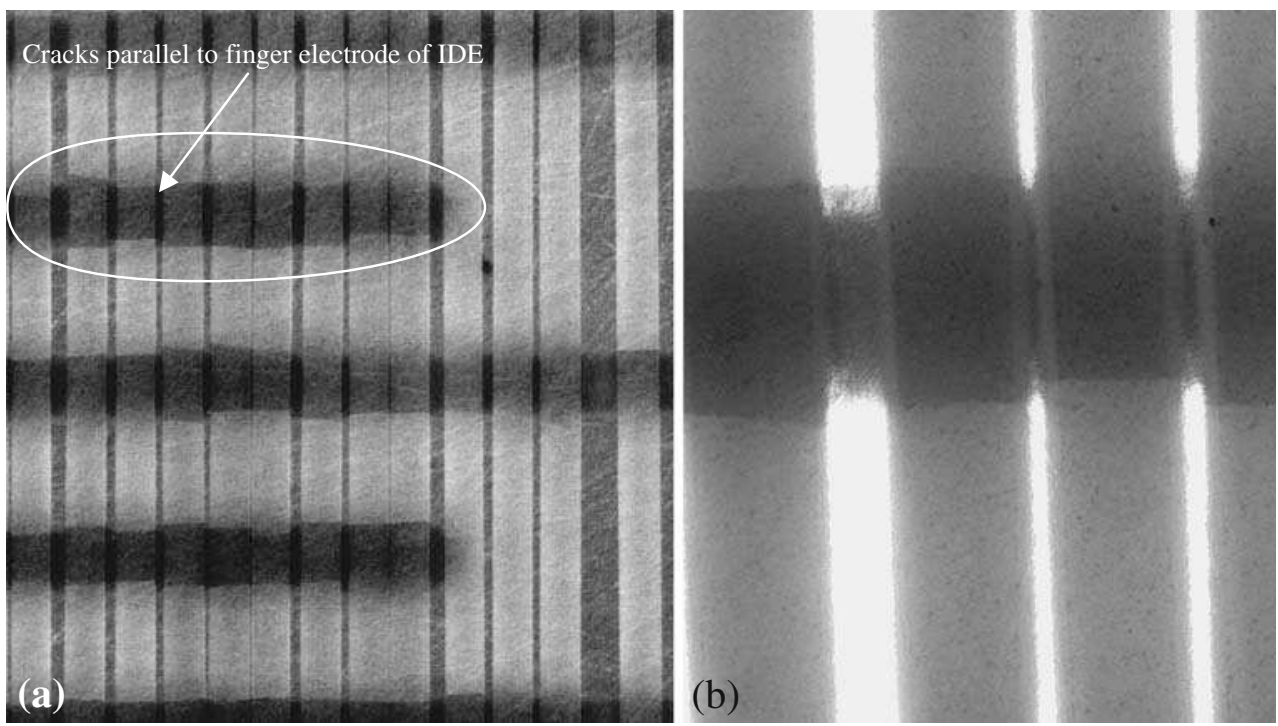


\section{References}

1. T. Tanimoto, K. Yamamoto, T. Morii, Nonlinear stress-strain behavior of piezoelectric ceramics under tensile loading, IEEE International Symposium on Applications of Ferroelectrics, 394397 (1994)

2. F. Fang, W. Yang, Poling-enhanced fracture resistance of lead zirconate titanate ferroelectric ceramics J. Mater. Sci. Lett. 46, 131 (2000). doi:10.1016/S0167-577X(00)00155-5

3. C.Q. Ru, X. Mao, M. Epstein, Electric-field induced interfacial cracking in multilayer electrostrictive actuators J. Mech. Phys. Solids 46, 1301 (1998). doi:10.1016/S0022-5096(98)00038-6

4. R.Q. Ye, L.H. He, Electric field and stresses concentrations at the edge of parallel electrodes in piezoelectric ceramics Int. J. Solids Struct. 38, 6941 (2001). doi:10.1016/S0020-7683(00)00398-X

5. A. Bent, N.W. Hagood, Piezoelectric fiber composites with interdigitated electrodes J. Intell. Mater. Syst. Struct. 8, 903 (1997)

6. R.B. Williams, D.J. Inman, M.R. Schultz, M.W. Hyer, W.K. Wilkie, Nonlinear tensile and shear behavior of macro fiber composite actuators J. Compos. Mater. 38, 855 (2004). doi:10.1177/0021998304040555

7. R. Paradies, M. Melnykowycz, Numerical stress investigation for piezoelectric elements with a circular cross section and interdigitated electrodes J. Intell. Mater. Syst. Struct. 18, 963 (2007). doi: $10.1177 / 1045389$ X06071438

8. C.R. Bowen, A. Bowles, S. Drake, N. Johnson, S. Mahon, Fabrication and finite element modelling of interdigitated electrodes Ferroelectrics 228, 257 (1999). doi:10.1080/0015019990 8226140
9. W. Beckert, W.S. Kreher, Modelling piezoelectric modules with interdigitated electrode structures Comput. Mater. Sci. 26, 36 (2003). doi:10.1016/S0927-0256(02)00390-7

10. C.R. Bowen, L.J. Nelson, R. Stevens, M.G. Cain, M. Stewart, Optimisation of interdigitated electrodes for piezoelectric actuators and active fibre composites J. Electroceram. 16, 263 (2006). doi:10.1007/s10832-006-9862-8

11. A.C. Dent, C.R. Bowen, R. Stevens, M.G. Cain, M. Stewart, Effective elastic properties for unpoled barium titanate J. Eur. Ceram. Soc. 27, 3739 (2007). doi:10.1016/j.jeurceramsoc.2007.02.031

12. M. Marutake, A calculation of physical constants of ceramic barium titanate J. Phys. Soc. Jpn. 11, 807 (1956). doi:10.1143/ JPSJ.11.807

13. T. Tanimoto, K. Okazaki, K. Yamamoto, Tensile stress-strain behavior of piezoelectric ceramics Jpn. J. Appl. Phys. 32, 4233 (1993). doi:10.1143/JJAP.32.4233

14. R. Paradies, Numerical investigation of piezoelectric actuators of the type AFC/MFC. Numerical Simulation of Electromechanical Systems, Proceedings NAFEMS Seminar 2005, Wiesbaden, Germany

15. R. Paradies, B. Schläpfer, Finite element modeling of piezoelectric elements with complex electrode configuration. Paper No. 60, Proceedings: 18th International Conference of Adaptive Structures and Technologies, 3-5 October 2007, Ottawa, 14 pp

16. N. Pini, S. Busato, H.R. Elsener, P. Ermanni, In situ growth of interdigitated electrodes made of polypyrrole for active fiber composites Polym. Adv. Technol. 18, 249 (2007). doi:10.1002/pat.883

17. M. Melnykowycz, X. Kornmann, C. Huber, M. Barbezat, A.J. Brunner, Performance of integrated active fiber composites in fiber reinforced epoxy laminates Smart Mater. Struct. 15, 204 (2006). doi:10.1088/0964-1726/15/1/050 